\title{
Out-of-the-box Universal Romanization Tool uroman
}

\author{
Ulf Hermjakob, Jonathan May, Kevin Knight \\ Information Sciences Institute, University of Southern California \\ \{ulf, jonmay, knight\}@isi.edu
}

\begin{abstract}
We present uroman, a tool for converting text in myriads of languages and scripts such as Chinese, Arabic and Cyrillic into a common Latin-script representation. The tool relies on Unicode data and other tables, and handles nearly all character sets, including some that are quite obscure such as Tibetan and Tifinagh. uroman converts digital numbers in various scripts to Western Arabic numerals. Romanization enables the application of string-similarity metrics to texts from different scripts without the need and complexity of an intermediate phonetic representation. The tool is freely and publicly available as a Perl script suitable for inclusion in data processing pipelines and as an interactive demo web page.
\end{abstract}

\section{Introduction}

String similarity is a useful feature in many natural language processing tasks. In machine translation, it can be used to improve the alignment of bitexts, and for low-resource languages with a related language of larger resources, it can help to decode out-of-vocabulary words. For example, suppose we have to translate degustazione del vino without any occurrence of degustazione in any training corpora, but we do know that in a related language dégustation de vin means wine tasting, we can use string similarity to infer the meaning of degustazione.

String similarity metrics typically assume that the strings are in the same script, but many crosslingual tasks such as machine translation often involve multiple scripts. If we can romanize text from a non-Latin script to Latin, standard string similarity metrics can be applied, including edit distance-based metrics (Levenshtein, 1966; Winkler, 1990) and phonetic-based metrics such as Metaphone (Philips, 2000).

Hindi, for example, is written in the Devanagari script and Urdu in the Arabic script, so any words between those two languages will superficially appear to be very different, even though the two languages are closely related. After romanization, however, the similarities become apparent, as can be seen in Table 1:

\begin{tabular}{|llcl|}
\hline & Hindi & Urdu & English \\
Original & नेपाल & نيl & Nepal \\
Romanization & nepaal & nipal & Nepal \\
\hline
\end{tabular}

Table 1: Example of Hindi and Urdu romanization

Foreign scripts also present a massive cognitive barrier to humans who are not familiar with them. We devised a utility that allows people to translate text from languages they don't know, using the same information available to a machine translation system (Hermjakob et al., 2018). We found that when we asked native English speakers to use this utility to translate text from languages such as Uyghur or Bengali to English, they strongly preferred working on the romanized version of the source language compared to its original form and indeed found using the native, unfamiliar script to be a nearly impossible task.

\subsection{Scope of Romanization}

Romanization maps characters or groups of characters in one script to a character or group of characters in the Latin script (ASCII) with the goal to approximate the pronunciation of the original text and to map cognates in various languages to similar words in the Latin script, typically without the 


\begin{tabular}{|c|c|c|}
\hline Amharic & 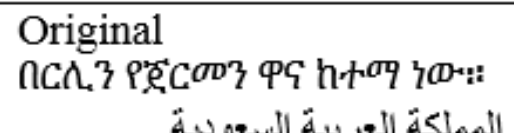 & $\begin{array}{l}\text { Romanization } \\
\text { bareline yajaremane waanaa katamaa nawe. }\end{array}$ \\
\hline Greek & 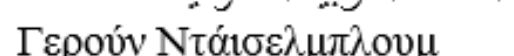 & Geroun Daiselbloum \\
\hline Hebrew & עזרת תורה בירושלים & 'zrt tvrh vyrvshlym \\
\hline Japanese & アメリカ & amerika \\
\hline Korean & $\begin{array}{l}\text { 세계에서 6번째로 면적이 } \\
\text { 넓은 나라이다. }\end{array}$ & $\begin{array}{l}\text { segyeeseo } 6 \text { beonjjaero myeonjeogi neolbeun } \\
\text { naraida. }\end{array}$ \\
\hline Mandarin & 北卡罗来纳 & beikaluolaina \\
\hline Nepali & $\begin{array}{l}\text { तिब्बती भाषामा यसको नाम } \\
\text { चोमोलुड्गमा हो। }\end{array}$ & $\begin{array}{l}\text { tibbatii bhaassaamaa yasako naam } \\
\text { comolunggamaa ho. }\end{array}$ \\
\hline Tamil & இதன் தலைநகராகச் & itan talainakaraakac cennai ullatu. \\
\hline Tibetan & 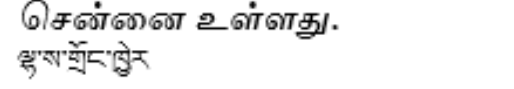 & lha'sa'grong $\cdot$ khyer \\
\hline
\end{tabular}

Table 2: Romanization examples for 10 scripts

use of any large-scale lexical resources. As a secondary goal, romanization standards tend to prefer reversible mappings. For example, as standalone vowels, the Greek letters ! (iota) and $v$ (upsilon) are romanized to $i$ and $y$ respectively, even though they have the same pronunciation in Modern Greek.

uroman generally follows such preference, but uroman is not always fully reversible. For example, since uroman maps letters to ASCII characters, the romanized text does not contain any diacritics, so the French word ou ("or") and its homophone où ("where") both map to romanized ou.

uroman provides the option to map to a plain string or to a lattice of romanized text, which allows the system to output alternative romanizations. This is particularly useful for source languages that use the same character for significantly different sounds. The Hebrew letter Pe for example can stand for both $p$ and $f$. Lattices are output in JSON format.

Note that romanization does not necessarily capture the exact pronunciation, which varies across time and space (due to language change and dialects) and can be subject to a number of processes of phonetic assimilation. It also is not a translation of names and cognates to English (or any other target language). See Table 3 for examples for Greek.

A romanizer is not a full transliterator. For example, this tool does not vowelize text that lacks explicit vowelization such as normally

\begin{tabular}{|llll|}
\hline Modern Greek & Kpń $\uparrow$ & $\gamma \varepsilon \omega \lambda \gamma^{\prime} \dot{\alpha} \alpha$ & $\mu \pi \alpha \nu \alpha \dot{\nu} \alpha$ \\
Pronunciation & Kriti & yeoloyia & banana \\
Romanization & Krete & geologia & banana \\
English & Crete & geology & banana \\
German & Kreta & Geologie & Banane \\
\hline
\end{tabular}

Table 3: Examples of Greek romanization

occurring text in Arabic and Hebrew (i.e., without diacritics/points); see Table 4.

\begin{tabular}{|c|c|c|}
\hline Arabic & $\begin{array}{c}\text { Normal text } \\
\text { giانطنن }\end{array}$ & 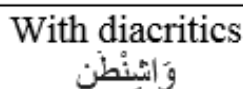 \\
\hline Romanization & washntn & waashintun \\
\hline English & Washington & Washington \\
\hline
\end{tabular}

Table 4: Romanization with and without diacritics

\subsection{Features}

uroman has the following features:

1. Input: UTF8-encoded text and an optional ISO-639-3 language code

2. Output: Romanized text (default) or lattice of romanization alternatives in JSON format

3. Nearly universal romanization ${ }^{1}$

4. N-to-m mapping for groups of characters that are non-decomposable with respect to romanization

5. Context-sensitive and source languagespecific romanization rules

\footnotetext{
${ }^{1}$ See Section 4 for a few limitations.
} 
6. Romanization includes (digital) numbers

7. Romanization includes punctuation

8. Preserves capitalization

9. Freely and publicly available

Romanization tools have long existed for specific individual languages such as the Kakasi ${ }^{2}$ kanji-to-kana/romaji converter for Japanese, but to the best of our knowledge, we present the first publicly available (near) universal romanizer that handles n-to-m character mappings. Many romanization examples are shown in Table 2 and examples of n-to-m character mapping rules are shown in Table 7 .

\section{System Description}

\subsection{Unicode Data}

As its basis, uroman uses the character descriptions of the Unicode table. ${ }^{3}$ For the characters of most scripts, the Unicode table contains descriptions such as CYRILLIC SMALL LETTER SHORT $U$ or CYRILLIC CAPITAL LETTER TE WITH $M I D D L E H O O K$. Using a few heuristics, uroman identifies the phonetic token in that description, i.e. $U$ and $T E$ for the examples above. The heuristics use a list of anchor keywords such as letter and syllable as well as a number of modifier patterns that can be discarded. Given the phonetic token of the Unicode description, uroman then uses a second set of heuristics to predict the romanization for these phonetic tokens, i.e. $u$ and $t$. For example, if the phonetic token is one of more consonants followed by one or more vowels, the predicted romanization is the leading sequence of consonants, e.g. SHA $\rightarrow$ sh.

\subsection{Additional Tables}

However, these heuristics often fail. An example of a particularly spectacular failure is SCHWA $\rightarrow s c h w$ instead of the desired $e$. Additionally, there are sequences of characters with noncompositional romanization. For example, the standard romanization for the Greek sequence omikron+upsilon, (ou) is the Latin ou rather than the character-by-character romanization $o y$.

As a remedy, we manually created additional correction tables that map sequences of one or more characters to the desired romanization, with

\footnotetext{
${ }^{2}$ http://kakasi.namazu.org

${ }^{3} \mathrm{ftp} / / / \mathrm{ftp}$.unicode.org/Public/UNIDATA/UnicodeData.txt
}

currently 1,088 entries. The entries in these tables can be restricted by conditions, for example to specific languages or to the beginning of a word, and can express alternative romanizations. This data table is a core contribution of the tool.

uroman additionally includes a few special heuristics cast in code, such as for the vowelizations of a number of Indian languages and Tibetan, dealing with diacritics, and a few languagespecific idiosyncrasies such as the Japanese sokuon and Thai consonant-vowel swaps.

Building these uroman resources has been greatly facilitated by information drawn from Wikipedia, ${ }^{4}$ Richard Ishida's script notes, ${ }^{5}$ and ALA-LC Romanization Tables. ${ }^{6}$

\subsection{Characters without Unicode Description}

The Unicode table does not include character descriptions for all scripts.

For Chinese characters, we use a Mandarin pinyin table for romanization.

For Korean, we use a short standard Hangul romanization algorithm. ${ }^{7}$

For Egyptian hieroglyphs, we added singlesound phonetic characters and numbers to uroman's additional tables.

\subsection{Numbers}

uroman also romanizes numbers in digital form.

For some scripts, number characters map oneto-one to Western Arabic numerals 0-9, e.g. for Bengali, Eastern Arabic and Hindi.

For other scripts, such as Amharic, Chinese, and Egyptian hieroglyphics, written numbers are structurally different, e.g. the Amharic number character sequence 10.9.100.90.8 = 1998 and the Chinese number character sequence $2 \cdot 10 \cdot 5 \cdot 10000 \cdot 6 \cdot 1000=256000$. uroman includes a special number module to accomplish this latter type of mapping. Examples are shown in Table 5.

Note that for phonetically spelled-out numbers

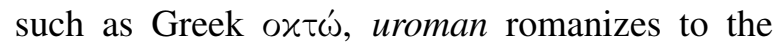
spelled-out Latin okto rather than the digital 8.

\footnotetext{
${ }^{4}$ https://en.wikipedia.org

${ }^{5}$ https://r12a.github.io/scripts/featurelist

${ }^{6} \mathrm{https}: / / \mathrm{www}$. loc.gov/catdir/cpso/roman.html

${ }^{7}$ http://gernot-katzers-spice-pages.com/var/

korean_hangul_unicode.html
} 


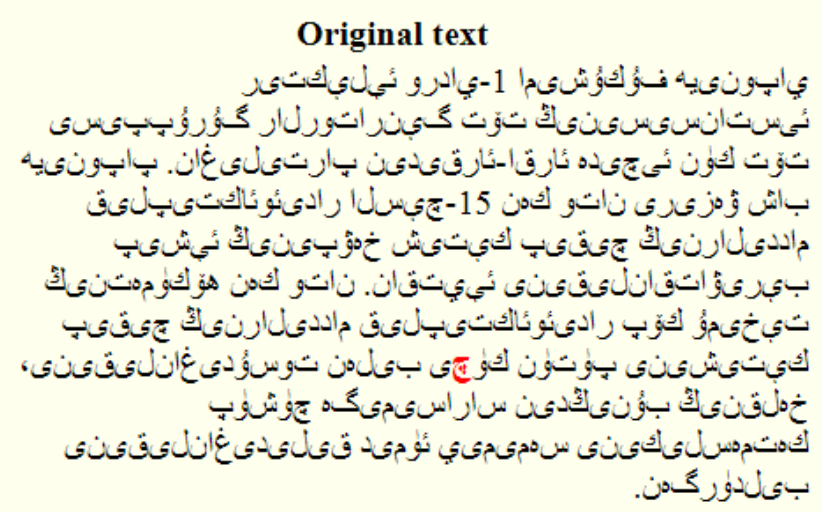

\section{Romanization}

yaponie fukushima 1-yadro elektir istansisining toet genratorlar guruppisi toet kuen ichide arqaarqidin partilighan. paponie bash weziri nato ken 15-chesla radioaktipliq maddilarning chiqip ketish xewpining eship beriwatqanliqini eytqan. nato ken hoekuemetning teximu koep radioaktipliq maddilarning chiqip ketishini puetuen kuechi bilen tosudighanliqini, xelqning buningdin sar Alternative romanizations: ch, q meslikini semimiy uemid qilidighanliqini bilduergen.

Figure 1: Screenshot of Uyghur romanization on demo site at bit.ly/uroman

\begin{tabular}{|lll|}
\hline \multirow{2}{*}{ Amharic } & Original & Romanization \\
Bengali & د৯8৯ & 1998 \\
Chinese & 二十五万六千 & 1949 \\
& 25.6万 & 256000 \\
\hline
\end{tabular}

Table 5: Romanization/Arabization of numbers

\subsection{Romanization of Latin Text}

Some Latin script-based languages have words for which spelling and pronunciation differ substantially, e.g. the English name Knight (IPA: /natt/) and French Bordeaux (/bor.do/), which complicates string similarity matching if the corresponding spelling of the word in the non-Latin script is based on pronunciation.

uroman therefore offers alternative romanizations for words such as Knight and Bordeaux (see Table 6 for an example of the former), but, as a policy uroman always preserves the original Latin spelling, minus any diacritics, as the top romanization alternative.

\begin{tabular}{|lll|}
\hline & Japanese & English \\
Original & ナイト & Knight \\
Top romanization & naito & Knight \\
An alternative rom. & nait & Nait \\
Alternative lattice & nai(to|t) & $(\mathrm{Kn} \mid \mathrm{N})$ (ight|ait) \\
\hline
\end{tabular}

Table 6: Romanization with alternatives

Table 7 includes examples of the Romanization rules in uroman, including n-to-m mappings.

\subsection{Caching}

uroman caches token romanizations for speed.

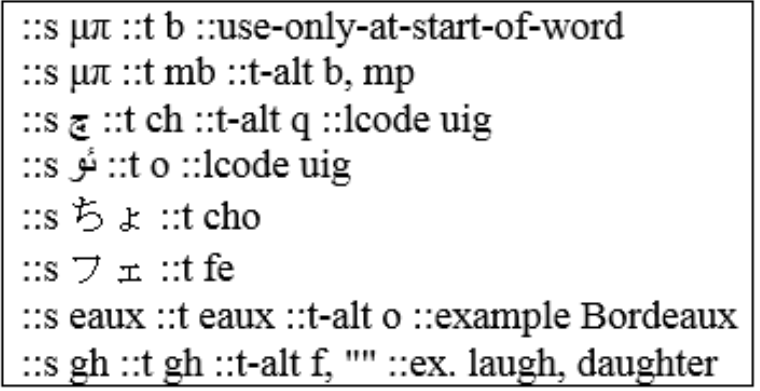

Table 7: Romanization rules with two examples each for Greek, Uyghur, Japanese, and English, with a variety of n-to-m mappings.

$(:: \mathrm{s}=$ source $;: \mathrm{t}=$ target$;::$ lcode $=$ language code $)$

\section{Download and Demo Sites}

uroman v1.2 is publicly available for download at bit.ly/isi-nlp-software. The fully self-sufficient software package includes the implementation of uroman in Perl with all necessary data tables. The software is easy to install (gunzip and tar), without any need for compilation or any other software (other than Perl).

Typical call (for plain text output):

uroman.pl --lc uig $<$ STDIN $>$ STDOUT

where $-l c$ uig specifies the (optional) language code (e.g. Uyghur).

There is also an interactive demo site at bit.ly/uroman. Users may enter text in the language and script of their choice, optionally specify a language code, and then have uroman romanize the text.

Additionally, the demo page includes sample texts in 290 languages in a wide variety of scripts. Texts in 21 sample languages are available on the demo start page and more are accessible as ran- 
dom texts. After picking the first random text, additional random texts will be available from three corpora to choose from (small, large, and Wikipedia articles about the US). Users can then restrict the randomness in a special option field. For example, --1 will exclude texts in Latin+ scripts. For further information about possible restrictions, hover over the word restriction (a dotted underline indicates that additional info will be shown when a user hovers over it).

The romanization of the output at the demo site is mouse sensitive. Hovering over characters of either the original or romanized text, the page will highlight corresponding characters. See Figure 1 for an example. Hovering over the original text will also display additional information such as the Unicode name and any numeric value. To support this interactive demo site, the uroman package also includes fonts for Burmese, Tifinagh, Klingon, and Egyptian hieroglyphs, as they are sometimes missing from standard browser font packages.

\section{Limitations and Future Work}

The current version of uroman has a few limitations, some of which we plan to address in future versions. For Japanese, uroman currently romanizes hiragana and katakana as expected, but kanji are interpreted as Chinese characters and romanized as such. For Egyptian hieroglyphs, only single-sound phonetic characters and numbers are currently romanized. For Linear B, only phonetic syllabic characters are romanized. For some other extinct scripts such as cuneiform, no romanization is provided.

uroman allows the user to specify an ISO-639-3 source language code, e.g. uig for Uyghur. This invokes any language-specific romanization rules for languages that share a script with other languages. Without source language code specification, uroman assumes a default language, e.g. Arabic for text in Arabic script. We are considering adding a source language detection component that will automatically determine whether an Arabic-script source text is Arabic, Farsi, or Uyghur etc.

\section{Romanization Applications}

\subsection{Related Work}

Gey (2009) reports that romanization based on ALA-LC romanization tables (see Section 2.2) is useful in cross-lingual information retrieval.

There is a body of work mapping text to phonetic representations. Deri and Knight (2016) use Wiktionary and Wikipedia resources to learn text-to-phoneme mappings. Phonetic representations are used in a number of end-to-end transliteration systems (Knight and Graehl, 1998; Yoon et al., 2007). Qian et al. (2010) describe the toolkit ScriptTranscriber, which extracts crosslingual transliteration pairs from comparable corpora. A core component of ScriptTranscriber maps text to an ASCII variant of the International Phonetic Alphabet (IPA).

Andy $\mathrm{Hu}^{\text {'s transliterator }}{ }^{8}$ is a fairly universal romanizer in JavaScript, limited to romanizing one Unicode character at a time, without context.

\subsection{Applications Using uroman}

Ji et al. (2017) and Mayfield et al. (2017) use uroman for named entity recognition. Mayhew et al. (2016) use uroman for (end-to-end) transliteration. Cheung et al. (2017) use uroman for machine translation of low-resource languages.

uroman has also been used in our aforementioned translation utility (Hermjakob et al., 2018), where humans translate text to another language, with computer support, with high fluency in the target language (English), but no prior knowledge of the source language.

uroman has been partially ported by third parties to Python and Java. ${ }^{9}$

\section{Conclusion}

Romanization tools have long existed for specific individual languages, but to the best of our knowledge, we present the first publicly available (near) universal romanizer that handles $n$-to-m character mappings. The tool offers both simple plain text as well as lattice output with alternatives, and includes romanization of numbers in digital form. It has been successfully deployed in a number of multi-lingual natural language systems.

\section{Acknowledgment}

This work is supported by DARPA (HR0011-15C-0115).

\footnotetext{
${ }^{8}$ https://github.com/andyhu/transliteration

${ }^{9} \mathrm{https}: / /$ github.com/BBN-E/bbn-transliterator
} 


\section{References}

Leon Cheung, Thamme Gowda, Ulf Hermjakob, Nelson Liu, Jonathan May, Alexandra Mayn, Nima Pourdamghani, Michael Pust, Kevin Knight, Nikolaos Malandrakis, et al. 2017. Elisa system description for LoReHLT 2017.

Aliya Deri and Kevin Knight. 2016. Grapheme-tophoneme models for (almost) any language. In Proceedings of the 54th Annual Meeting of the Association for Computational Linguistics (Volume 1: Long Papers), volume 1, pages 399-408.

Fredric Gey. 2009. Romanization-an untapped resource for out-of-vocabulary machine translation for CLIR. In SIGIR Workshop on Information Access in a Multilingual World, pages 49-51.

Ulf Hermjakob, Jonathan May, Michael Pust, and Kevin Knight. 2018. Translating a language you don't know in the Chinese Room. In Proceedings of the 56th Annual Meeting of Association for Computational Linguistics, Demo Track.

Heng Ji, Xiaoman Pan, Boliang Zhang, Joel Nothman, James Mayfield, Paul McNamee, and Cash Costello. 2017. Overview of TAC-KBP2017 13 languages entity discovery and linking. In Text Analysis Conference - Knowledge Base Population track.

Kevin Knight and Jonathan Graehl. 1998. Machine transliteration. Computational Linguistics, 24(4):599-612.

Vladimir I Levenshtein. 1966. Binary codes capable of correcting deletions, insertions, and reversals. In Soviet physics doklady, volume 10, pages 707-710.

James Mayfield, Paul McNamee, and Cash Costello. 2017. Language-independent named entity analysis using parallel projection and rule-based disambiguation. In Proceedings of the 6th Workshop on BaltoSlavic Natural Language Processing, pages 92-96.

Stephen Mayhew, Christos Christodoulopoulos, and Dan Roth. 2016. Transliteration in any language with surrogate languages. arXiv preprint arXiv:1609.04325.

Lawrence Philips. 2000. The double Metaphone search algorithm. C/C++ Users J., 18(6):38-43.

Ting Qian, Kristy Hollingshead, Su-youn Yoon, Kyoung-young Kim, and Richard Sproat. 2010. A Python toolkit for universal transliteration. In LREC.

William E. Winkler. 1990. String comparator metrics and enhanced decision rules in the Fellegi-Sunter model of record linkage. In Proceedings of the Section on Survey Research Methods, pages 354-359. ERIC.
Su-Youn Yoon, Kyoung-Young Kim, and Richard Sproat. 2007. Multilingual transliteration using feature based phonetic method. In Proceedings of the 45th annual meeting of the Association of Computational Linguistics, pages 112-119. 